\title{
Topology Optimization of Structure with Asymmetry Cementitious Granular Material
}

\author{
Haipeng $\mathrm{Jia}^{1 *}$, Qunying Men ${ }^{1}$, Anil Misra ${ }^{2}$ and Congyi Liu ${ }^{1}$ \\ ${ }^{1}$ Department of Mechanical Engineering, Hebei University of Technology Tianjin, 300130, P. R. China \\ ${ }^{2}$ Civil, Environmental and Architectural Engineering Department, Bioengineering Research Center (BERC), University of Kansas, \\ 1530 W. 15th Street, Learned Hall, Lawrence, KS 66045-7609, USA \\ ${ }^{*}$ Corresponding author
}

\begin{abstract}
Topology optimization of structure of cementitious granular material with different tension and compression properties is one of challenging and important branches in engineering application for the complex stress and strain relationship of cohesive material. Applying evolutionary structure optimization technique, this paper proposes the mathematical formulation and computational algorithm of structure topology optimization of cohesive granular material based on the tension-compression asymmetry constitutive equation. The objective function is to minimize the mean compliance of structure. Numerical example illustrated the effectiveness of the proposed algorithm.
\end{abstract}

Keywords-granular cementitious material; tension-compression asymmetry; structure topology optimization; evolutionary structure optimization

\section{INTRODUCTION}

Topology optimization has become increasingly popular in industrial application during the last decades for tremendous cost savings can be obtained for its impact in the early stage of structure design. Research in this fields has been one of the most challenging topics in structure optimization, and much achievement has been gotten not only in theory development and engineering application (Eschenauer, 2001; Rozvany, 2009; Joshua, 2014). Topology optimization technique saw widespread applications in engineering design and much received benefit put the research work forward considerably. It still exits a bottle neck that design optimal structure like that of the bio-structure, though engineer get more hints from nature structure through bionomics.

Bendsøe \& Kikuchi (1988) proposed the homogenization method for structure topology optimization. Topology optimization methods based on element-wise design variables, such as the Solid Isotropic Material with penalization (SIMP) method (Bendsøe and Sigmund 2003) and Evolutionary Structural Optimization (ESO) methods (Huang and Xie 2010). Originally developed as a mathematical numerical simulation tool for tracking the motion of interfaces in two or three dimensions, the level set method (see papers in Wang et al. 2003; Allaire et al. 2004 and 2005; Ullah et al. 2013; Dunning et al 2015; Jia 2011) was introduced into the topology optimization. Using bar element, Peng (2014) proposed a stiffness spreading method for topology optimization of truss structure. This apprach can be optimize the size, shape and topology of truss structures simultaneously.
For the symmetric prescribed loading and support conditions, Rozvany and Xu (Rozvany 2011; Xu 2012, 2013) found that many structural optimization problems that can be formulated as convex programs and there exists at least one symmetric global optimal solution. This is only effective for the isotropic material.

But most papers and industrial applications are focus on continuum material model with given elastic tensor, and little literatures is related with granular material whose constitutive equation depends on the microstructure and their relationship, in which the tension-compression asymmetry can be considered (Misra, 2015).

Cohesive granular material and its structure play important roles in engineering application and biomaterial synthesis. The key problem is to find the relationship of stress and strain during the updating within optimization and topology optimization. This paper proposes the computational algorithm and its implementation of this kind of topology optimization problems.

\section{STRUCTURE TOPOLOGY OPTIMIZATION USING GRANULAR MICROMECHANICS}

Based on the tension-compression asymmetry constitutive equation of cohesive granular material. This paper proposes the mathematical formulation and computational algorithm of structure topology optimization of cohesive granular material.

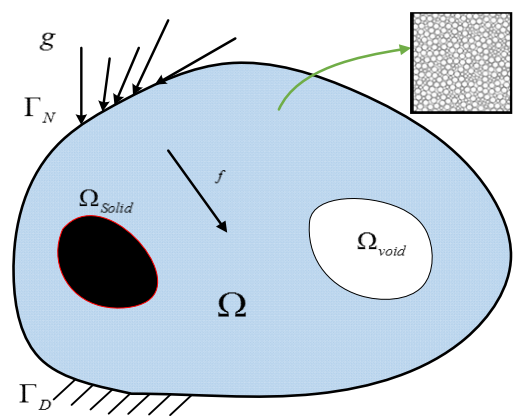

FIGURE I. DESIGN DOMAIN FOR TOPOLOGY OPTIMIZATION OF COHESIVE GRANULAR MATERIAL AND ITS BOUNDARY CONDITIONS

Results show that the material model can be used to design the tension-compression asymmetry structure, which cannot be 
implemented for the traditional material constitutive relationship.

Let $\Omega$ be a bounded open set occupied by an elastic material as shown in figure 1. The boundary of $\Omega$ is made of two disjoint parts $\left(\Gamma_{D}\right.$ and $\left.\Gamma_{N}\right)$. Let us assume that we have Dirichlet boundary conditions on $\Gamma_{D}$, and Neumann boundary conditions on $\Gamma_{N}$. All admissible topology are required to be a subset of a design domain $\Omega$. We denote by $f$ the vectorvalued function of the volume forces and by $g$ that of the surface valued function of external loadings. The displacement field $u$ in $\Omega$ is thus the solution of following system of equations.

$$
\begin{cases}-\operatorname{div}(A e(u))=f, & \text { in } \Omega \\ u=u_{0}, & \text { on } \Gamma_{D} \\ (A e(u))=g, & \text { on } \Gamma_{N}\end{cases}
$$

where the first equation is the equilibrium equation and second and third equations represent Dirichlet and Neumann boundary conditions respectively. The objective of the topology optimization of design domain, $\Omega$, is to find the function

$$
\chi(x)=\left\{\begin{array}{lll}
1 & \text { if } & x \in \Omega_{s} \\
0 & \text { if } & x \in \Omega / \Omega_{s}
\end{array} .\right.
$$

where $\mathrm{x}$ denotes design variables. We now consider the finite element formulation for the solution of Eq. (1).

For Cohesive granular material, as in figure 2, the relative displacement $\delta_{i}$ between two nearest neighbor grains $n$ and $p$ ignoring the grain rotations is written as

$$
\delta_{i}=u_{i}^{p}-u_{i}^{n}
$$

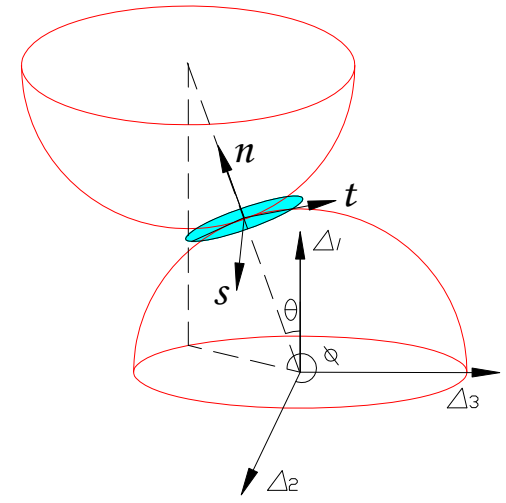

FIGURE II. THE RELATIONSHIP OF EACH PAIR OF GRAINS

Where, $\mathrm{u}_{\mathrm{i}}$ is the particle displacement; superscripts refer to the interacting particles. To obtain the continuum description of this discrete model, we utilize the Taylor series expansion of the displacement such that the displacement of grain $p$ may be written as:

$$
u_{i}^{p}=u_{i}^{n}+u_{i, j}^{n}\left(x_{j}^{p}-x_{j}^{n}\right)
$$

where, the point of expansion is chosen as the centroid of grain $n$. In Eq. 2, $u_{i, j}$ is the displacement gradient, and $x_{j}^{n}$ and $x_{j}^{p}$ are the position vectors of the centroids of grains $n$ and $p$, respectively. Thus, the relative displacement, $\delta_{i}$, between two nearest neighbor grains $n$ and $p$ representing the $\alpha$-th intergranular interaction is given by

$$
\delta_{i}^{\alpha}=u_{i, j}^{n}\left(x_{j}^{p}-x_{j}^{n}\right)=u_{i, j}^{n} l_{j}^{\alpha}=\varepsilon_{i j} l_{j}^{\alpha}
$$

where $l_{j}$ is an inter-granular branch vector joining the centroids of grains $n$ and $p$. So it is seen that up to here, the relative displacement of two grains inside the material is derived as the projection of the macroscopic strain tensor in the direction of the inter-granular interaction vector. This displacement vector is further decomposed into the directions of a local coordinate system defined separately for each grain-pair interaction. The local coordinates system is composed of one normal axis, $n$, in the direction of the vector joining the two particle centroids and two orthogonal axes lying in the tangential plane whose normal vector is n. Two grains and the direction of their local coordinate axes with respect to the global coordinate system can be seen in Figure 2.

The components of these axes are derived as

$$
\begin{aligned}
& n_{i}=\langle\cos \theta, \sin \theta \cos \phi, \sin \theta \sin \phi\rangle \\
& s_{i}=\langle-\sin \theta, \cos \theta \cos \phi, \cos \theta \sin \phi\rangle \\
& t_{i}=\langle 0,-\sin \phi, \cos \phi\rangle
\end{aligned}
$$

Using the defined coordinate system, the displacement vector, $\delta_{\mathrm{i}}$, is decomposed into components along the $\mathbf{n}, \mathbf{s}$, and $\mathbf{t}$ coordinate axes as follows

$$
\begin{aligned}
& \delta_{n}^{\alpha}=\delta_{i}^{\alpha} n_{i}^{\alpha} \\
& \delta_{s}^{\alpha}=\delta_{i}^{\alpha} s_{i}^{\alpha} \\
& \delta_{t}^{\alpha}=\delta_{i}^{\alpha} t_{i}^{\alpha}
\end{aligned}
$$

Conjugate to this displacement components, an interparticle force components is defined which is obtainable using a simple force law in the inter-particle scale. Normal and tangential stiffness coefficients are used to define the normal and tangential force components.

$$
\begin{aligned}
& k_{n}^{T}=\alpha k_{n} ; k_{s}^{T}=\alpha k_{s} ; \quad \text { for } \delta_{n}>0 . \\
& k_{n}^{C}=k_{n} ; \quad k_{s}^{C}=k_{s} ; \quad \text { for } \delta_{n} \leq 0 \text {. }
\end{aligned}
$$


In which, the superscript $\mathrm{T}$ and $\mathrm{C}$ denote tension and compression, respectively, and $\alpha$ is a constant between 0 and 1. In order to write the force components in the global coordinate system, rotation tensor is defined as follows for each inter-granular contact

$$
T=\left[\begin{array}{lll}
n_{1} & s_{1} & t_{1} \\
n_{2} & s_{2} & t_{2} \\
n_{3} & s_{3} & t_{3}
\end{array}\right]
$$

Thus, the inter-granular force-displacement relationship can be written as

$$
f_{i}=K_{i j} \delta_{j}
$$

In which,

$$
K=T \times k \times T^{T}
$$

The principle of virtual work is applied to all of the grainpairs and that done by the stress and strain tensors and using Eq. 5, one will get

$$
\begin{gathered}
\sigma_{i j} \varepsilon_{i j}=\frac{1}{V} \sum_{\alpha}\left(f_{i}^{\alpha} \delta_{i}^{\alpha}\right)=\left(\frac{1}{V} \sum_{\alpha}\left(f_{i}^{\alpha} l_{j}^{\alpha}\right)\right) \varepsilon_{i j} . \\
\sigma_{i j}=\frac{1}{V} \sum_{\alpha}\left(f_{i}^{\alpha} l_{j}^{\alpha}\right) .
\end{gathered}
$$

Now substituting Eq. 9 and then Eq. 5 into Eq. 10 one would derive the stress tensor in terms of strain tensor and the stiffness tensor.

$$
\sigma_{i j}=\left(\frac{1}{V} \sum_{\alpha}\left(K_{i k} l_{l}^{\alpha} l_{j}^{\alpha}\right)\right) \varepsilon_{k l}=C_{i j k l} \varepsilon_{k l} .
$$

Thus it is clearly seen that the stiffness tensor is derived using the local stiffness parameters as

$$
C_{i j k l}=\frac{1}{V} \sum_{\alpha}\left(K_{i k} l_{l}^{\alpha} l_{j}^{\alpha}\right)
$$

Based on the theory of cohesive granular material, the formulation of topology optimization of this kind material structure can be formulated as in Eq. 16.

$$
\begin{aligned}
& \text { Minimize: } J(u)=\frac{1}{2} u^{T} K u=\frac{1}{2} \sum_{e=1}^{N} u^{e T} k^{e} u^{e} . \\
& \text { Subject to: } a(u, v)=L(v) .
\end{aligned}
$$

$$
\begin{gathered}
\left.u\right|_{\Gamma_{D}}=u_{0} \forall v \in U . \\
V=\int d \Omega \leq V_{\max } .
\end{gathered}
$$

Evolutionary Structure Optimization (ESO) algorithm is based on the concept of gradually removing unnecessary or inefficient material from a structure, or gradually adding material at high stress area, to achieve an optimal design. The objective is to find the minimization of compliance of the structure, with constraint the volume of availability of material. Criteria are the strain energy of each element.

\section{NUMERICAL EXAMPLES}

The Cantilever problem is one of the typical structure topology optimization problems. The design objective is to minimize strain energy while satisfying volume constraints. The design domain and boundary condition is illustrated in Figure 3. Left edge is fixed to support structure and concentrated force $\mathrm{P}=1.0 \mathrm{~N}$ is applied at the middle point of right edge in the downward direction. In this example, the volume constraint is set at $50 \%$ of the total design domain, and the value indicating the extent tension-compression asymmetry is 0.5 . The design domain is discretized with $80 \mathrm{X} 60$ four node quadrilateral isoparametric plane stress element.

For the cantilever beam in Figure 3, it is a benchmark problem for topology optimization. For isotropic materials structure, the final topology should be symmetry for the symmetrical structure and boundary conditions.

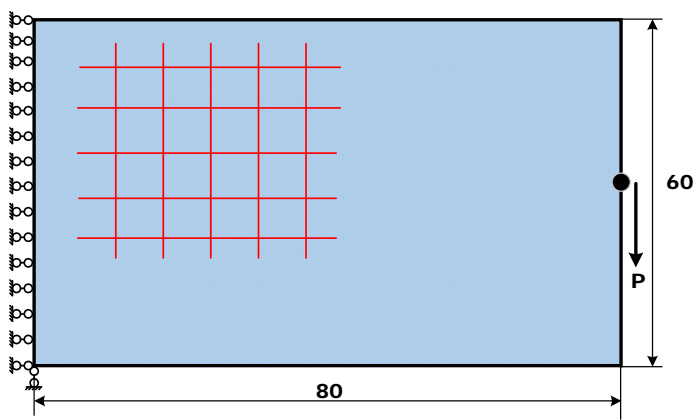

FIGURE III. GEOMETRY PARAMETERS AND BOUNDARY CONDITIONS OF CANTILEVER

In figure 4, the key steps for topology, figures $4 \mathrm{a}$ through $4 \mathrm{c}$ are given to demonstrate the topology variation. The first principle stress fields is computed to indicate the tension region in red and compression in blue. The effect of $\alpha$ on the topology is studied to show different tension-compression asymmetry of optimal topology in figure 5. Furthermore, the first principle stress given in figure 5 indicates the regions under tension in red and compression in blue. We can clearly see that the stiffer compressive regions are of larger size than the less stiff tensile regions. With the increase of $\alpha$, material transmitted from compression region to tension region, from asymmetry to symmetry with $\alpha=1.0$. Such a result is expected, since stiffer regions can support larger proportion of the applied loading. 


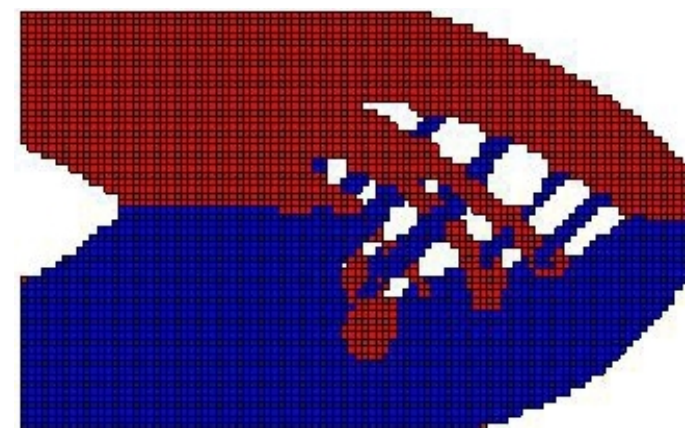

a) ITERATION NUMBER 20

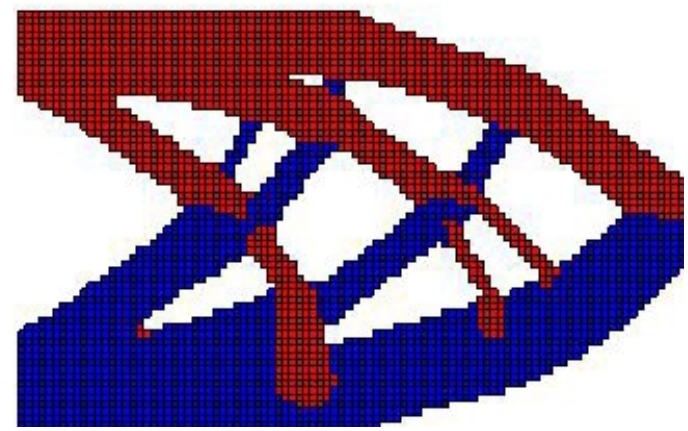

b) ITERATION NUMBER 53

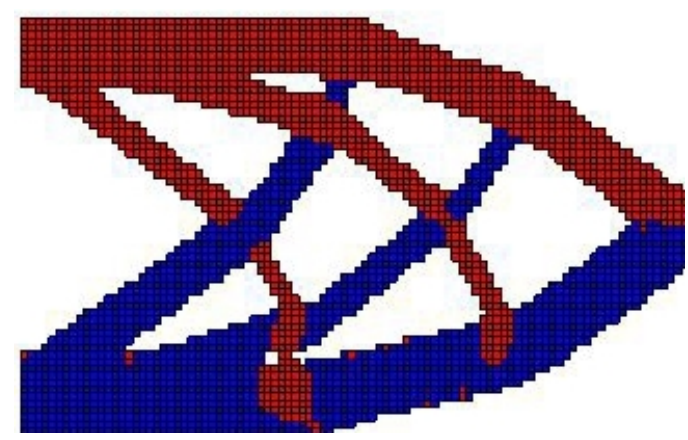

c) ITERATION NUMBER 77

FIGURE IV. TOPOLOGY VARIATION HISTORY WITH INTERMEDIATE KEY OPTIMIZATION ITERATION 20, 53, AND CONVERGED AT 77

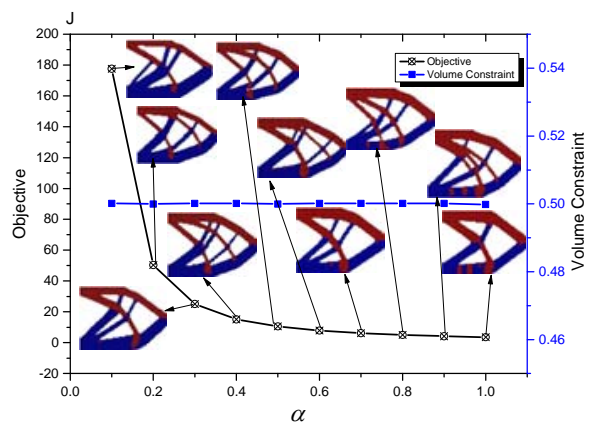

FIGURE V. TOPOLOGY VARIATION WITH $\alpha$, INCLUDING VALUE OF OBJECTIVE FUNCTION TOPOLOGY VARIATION, RED AND BLUE INDICATE TENSILE AND COMPRESSIVE MAJOR PRINCIPAL STRESS REGIONS, RESPECTIVELY

\section{CONCLUDING REMARKS}

This paper proposes the mathematical formulation and computational algorithm of structure topology optimization of cohesive granular material based on the tension-compression asymmetry constitutive equation. Numerical benchmark problem in structure topology optimization cantilever beam is computed and the asymmetry of final topology illustrated the validity and effectiveness of the proposed algorithm. The optimal topology variation is given, and the effect of ratio of tension and compression on the optimum topology is analyzed. The topology variation shows that of the proposed mathematical formulation for granular material structure topology optimization is computationally feasible.

\section{ACKNOWLEDGMENT}

This work was supported partially by National Science Foundation of China (51475137 and 51605135) and key project from Hebei Education Bureau (QN20131097). Their financial contribution is gratefully acknowledged.

\section{REFERENCES}

[1] Eschenauer HA, Olhoff N, "Topology optimization of continuum structures: a review”, Appl. Mech. Rev. 54(2001)331-390.

[2] G. I. N. Rozvany, "A critical review of established methods of structural topology optimization”, Struct Multidiscipl Optim. 37(2009)217-237.

[3] Joshua D. Deaton, Ramana V. Grandhi, "A survey of structural and multidisciplinary continuum topology optimization: post 2000", Struct Multidiscipl Optim. 49(2014)1-38.

[4] Bendsøe MP, Kikuchi N, "Generating optimal topologies in structura design using a homogenization method", Comput. methods Appl. Mech. Eng. 71(1988)197-224

[5] Michael Yu Wang, Xiaoming Wang, Dongming Guo, "A level set method for structural topology optimization", Comput. methods Appl. Mech. Eng. 192(2003)227-246.

[6] Allaire G, Jouve F., "A level-set method for vibration and multiple loads structural optimization”, Comput. methods Appl. Mech. Eng. 194(2005)3269-3290.

[7] Dunning, P.D. \& Kim, H.A., "A new hole insertion method for level se based topology optimization", International Journal for Numerical Methods in Engineering, 93(2013)118-134.

[8] Svanberg K, "The method of moving asymptotes-a new method for structural optimization", Int J Numer Meth Eng. 24(1987)359-373.

[9] Bruyneel M., Duysinx P., Fleury C. "A family of MMA approximation for structural optimization”, Struct Multidiscipl Optim. 24(2002)263-276.

[10] Ullah, B. and Trevelyan, J, "'Correlation between hole insertion criteria in a boundary element and level set based topology optimisation method, Eng Anal Bound Elem. 37(2013)1457-1470.

[11] Zhou K, "Topology optimization of truss-like continuum structures for natural frequencies", Struct Multidiscipl Optim. 47(2013) 613-619.

[12] Huang X, Xie YM, "Evolutionary topology optimization of continuum structures with an additional displacement constraint", Struct Multidiscipl Optim. 40(2010)409-416.

[13] Rozvany GIN., "On symmetry and non-uniqueness in exact topology optimization”, Struct. Multidiscipl. Optim. 43(2011)297-317.

[14] Xu Guo, Changhui Ni, Gengdong Cheng, Zongliang Du, "Some symmetry results for optimal solutions in structural optimization", Struct Multidiscipl. Optim., 2012, 46(2012)631-645.

[15] Xu Guo, Zongliang Du, Gengdong Cheng, Changhui Ni, "Symmetry properties in structural optimization-some extensions". Struct. Multidiscipl. Optim. 47(2013)783-794.

[16] Peng Wei, H. Ma, M. Y. Wang, "The stiffness spreading method for layout optimization of truss structures", Struct Multidiscipl Optim., 49 (2014)667-682. 
[17] Haipeng Jia, H.G. Beom, Yuxin Wang, Song Lin, Bo Liu. "Evolutionary level set method for structural topology optimization", Comput Struct. 89(2011)445-454.

[18] Anil Misra, Payam Poorsolhjouy, "Micro-macro scale instability in 2D regular granular assemblies", Continuum Mech Therm. 27(1-2)(2015) $63-82$ 